\title{
细胞外囊泡的流式检测 \\ Flow Cytometric Analysis of Extracellular Vesicles
}

闵娟, 关武祥*

中国科学院武汉病毒研究所, 武汉, 湖北省

*通讯作者邮箱: guanwx@wh.iov.cn

引用格式: 闵娟, 关武祥. (2019). 细菌外襄泡的流式检测. Bio-101 e1010319. Doi: 10.21769/BioProtoc.1010319.

How to cite: Min, J. and Guan, W. X. (2019). Flow Cytometric Analysis of Extracellular Vesicles. Bio-101 e1010319. Doi: 10.21769/BioProtoc.1010319. (in Chinese)

摘要: 细胞外囊泡 (extracellular vesicles, EVs) 是指由细胞分泌或者从细胞膜上脱落 的脂质双分子层囊泡状小体。根据 EVs 生物来源、性质及功能通常被分为外泌体 (exosomes)、微囊泡 (Microvesicles/microparticles) 和调亡小体 (Apoptosis body)。 $E V s$ 不仅携带母体细胞多种特征性生物信息分子，而且可以将这些分子传递给其他细胞， 在许多生理病理学过程中充当生物信息传递载体。因此, 对 EVs 进行准确的定性及定 量检测显得尤为重要。流式细胞仪理论上能对 EVs 进行高通量、快速及多参数的检测, 是目前 $E V s$ 检测的主要手段。本方案以循环微囊泡 (外周血中的微囊泡) 为例, 通过电 穿孔技术将超小锰磁性近红外量子点 $\left(\mathrm{Ag}_{2} \mathrm{Se} @ \mathrm{MnQDs}\right)$ 载入循环微囊泡, 利用流式细 胞仪对其进行检测。

关键词: 细胞外囊泡, 量子点, 标记, 流式细胞仪

\section{材料与试剂}

1. 含 EDTA-K2 抗凝剂真空采血管 (碧迪公司)

2. 电穿孔缓冲液 (北京百奥莱博科技有限公司)

3. 蔗糖 (Sigma-Aldrich)

4. 盐酸

5. 磷酸盐缓冲液 $(1 \times$ PBS) (见溶液配方)

6. $\mathrm{NaOH}(0.1 \mathrm{M})$ 溶液 (见溶液配方) 


\section{仪器设备}

1. 台式离心机 (Eppendorf, model: Centrifuge 5810R)

2. 超高速离心机 (Beckman coulter, model: Avanti J-26 XP)

3. 流式细胞仪 (BD, model: FACS AriallI)

4. 电穿孔仪 (Bio-Rad, model: Gene Pulser Xcell ${ }^{\mathrm{TM}}$ Bio-Rad)

\section{软件}

1. FlowJo 软件

\section{实验步骤}

一、循环微囊泡的提取与荧光标记 (详见图 1)

1. 用含 EDTA-K2 抗凝剂的真空采血管收集口腔癌患者 $5 \mathrm{ml}$ 静脉血样品。

2. 将样品转移至离心管中, $4{ }^{\circ} \mathrm{C}$ 条件下, $1,500 \times g$ 离心 $20 \mathrm{~min}$, 取上清。

3. 重复步骤 2 , 取上清, 加入上清体积 3 倍的无菌 PBS 稀释所得血清。

4. $4{ }^{\circ} \mathrm{C}$ 条件下 $50,000 \times g$ 离心 $60 \mathrm{~min}$, 以无菌 PBS 重悬管底沉淀。

5. 重复步骤 4 , 以 $150 \mu \mathrm{l}$ 无菌 PBS 重悬沉淀, 即获得口腔癌患者循环微囊泡。

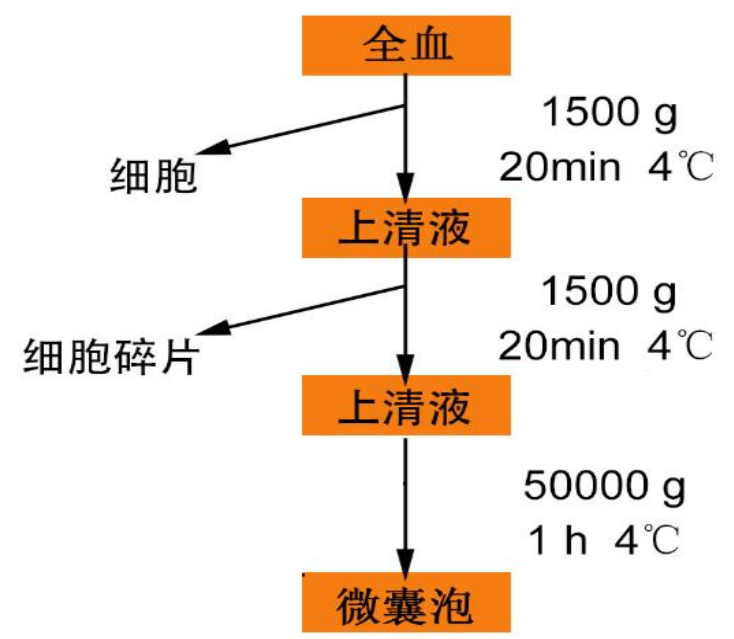

图 1. 循环微襄泡的提取

二、电穿孔辅助 $\mathrm{Ag}_{2} \mathrm{Se} @ \mathrm{Mn}$ 量子点标记循环微囊泡及其流式细胞仪检测 
1. 将 $20 \mu \mathrm{g}(50 \mu \mathrm{l})$ 循环微囊泡, $50 \mu \mathrm{l} \mathrm{Ag}$ Se@MnQDs $(1 \mathrm{mg} / \mathrm{ml})$ 和 $150 \mu \mathrm{l}$ 电穿孔缓 冲液加至电击杯 $(4 \mathrm{~mm})$ 中混匀。

2. 设置电穿孔参数: 输出脉冲电压 $250 \mathrm{~V}$, 电容 $250 \mu \mathrm{F}$, 单次脉冲。

3. 将电击杯置于电穿孔仪上, 按上述参数进行电穿孔。

4. 电穿孔结束后, 将混合液在 $37^{\circ} \mathrm{C}$ 下放置 $30 \mathrm{~min}$ 以促进微囊泡膜修复。

5. 以蔗糖密度梯度 (15\% 60\%) 离心去除游离量子点, 最后收集微囊泡层。

6. $4{ }^{\circ} \mathrm{C}$ 条件下 $50,000 \times g$ 离心 $60 \mathrm{~min}$, 以 $400 \mu \mathrm{lPBS}$ 重悬沉淀, 即获得 $\mathrm{Ag}_{2} \mathrm{Se} @ \mathrm{Mn}$ QD 标记的循环微囊泡。

三、流式细胞仪分析

1. 用 $A$ 管 (未加 $A_{2} \operatorname{Se} @ M n$ QD 的循环微囊泡) 设置 FSC-A 和 SSC-A 的初始值, 阈值设置为 FCS and SSC, 根据具体情况设置阈值大小, 用 FSC-A 和 FSC-H 排 除黏连囊泡, 使 $\mathrm{A}$ 管的荧光强度位于 $10^{0} \sim 10^{1}$ 。

2. 用 B 管 (在循环微囊泡加入 $A g_{2} S e @ M n Q D$, 但未电击处理), 检测 B 管的苂光强 度。

3. 用 $\mathrm{C}$ 管 (在循环微囊泡加入 $\mathrm{Ag}_{2} \mathrm{Se} @ M n \mathrm{QD}$, 并电击处理), 检测 C 管的荧光强度。

4. 收集剩余样品, 如果个别样品荧光强度超出上限, 则需减小荧光通道电压至峰图在 可测范围内。

\section{结果与分析}

目前, 通过体外培养细胞获取微囊泡, 存在诸多局限和安全隐患, 体液来源的微囊泡则 具有许多天然优势 (Kanada 等, 2015; Pitt 等, 2016)。目前对于体液来源的微囊泡 的可视化标记主要依赖于传统荧光染料, 但这些染料自身存在光漂白、潜在的细胞毒性、 弱苂光信号、非特异性标记等先天缺陷 (Resch-Genger 等, 2008)。本方案旨在通过电 穿孔的策略将超小、近红外磁性量子点载入循环微囊泡, 利用流式细胞仪实现微囊泡的 定量检测 (Yu 等, 2017)。

收集口腔癌患者的静脉血, 差速离心法收集血液中循环微囊泡并重悬于无菌 PBS 中。将微囊泡与 $\mathrm{Ag}_{2} \mathrm{Se} @ \mathrm{Mn}$ 量子点和电穿孔缓冲液混匀后, 电击混合液。整个电击过 程仅需 40 50 ms。 


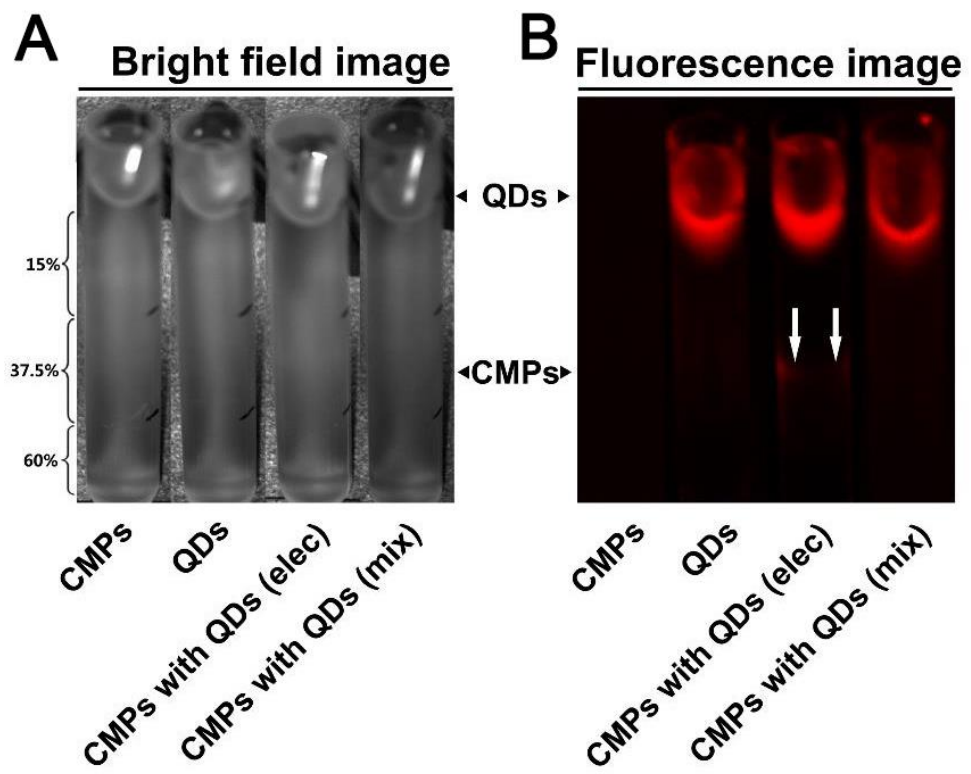

图 2. 蔗糖密度梯度离心法去除多余的量子点

收集混合液体, 以蔗糖密度梯度离心法去除多余量子点 (图 2), 电穿孔处理后的微 囊泡层可见 $\mathrm{Ag}_{2} \mathrm{Se} @ \mathrm{Mn}$ 量子点苂光信号, 初步证明电穿孔法可以用于循环微囊泡的 $\mathrm{Ag}_{2} \mathrm{Se} @ M n$ 量子点标记。获取微囊泡层, 超速离心法收集 $\mathrm{Ag}_{2} \mathrm{Se} @ M n$ 量子点标记的微 囊泡。

流式细胞术分析结果显示, 电穿孔法成功的将 $\mathrm{Ag}_{2} \mathrm{Se} @ \mathrm{Mn}$ 量子点载入循环微囊泡 (图 3A), 效率高达 90\%以上 (图 3B)。

A
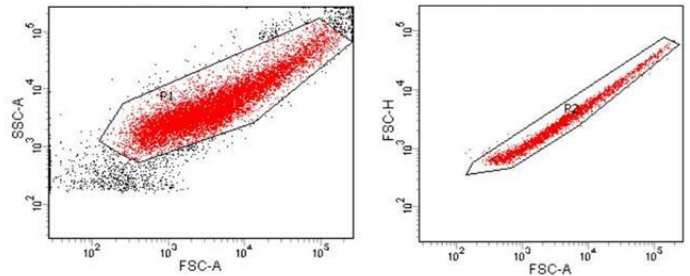

B

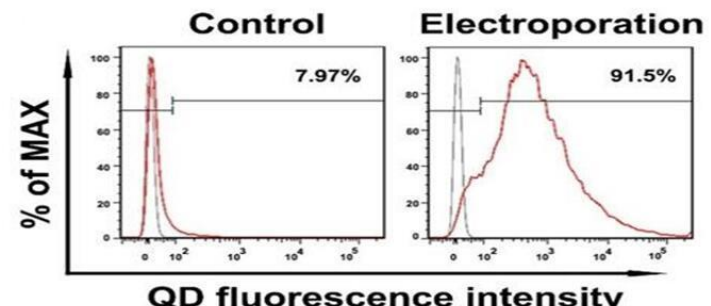

图 3 .电穿孔辅助 $\mathrm{Ag}_{2} \mathrm{Se} @ M n$ 量子点标记循环微囊泡. A.流式细胞分析微囊泡。B 流式细胞术分析微囊泡 $\mathrm{Ag}_{2} \mathrm{Se} @ M n$ 量子点标记效率。 


\section{失败经验}

1. 电穿孔 (Electroporation) 也叫电转染, 是通过高强度的电场作用, 在细胞膜上形 成直径约 $10 \mathrm{~nm}$ 的小孔, 细胞外的物质通过小孔进入胞内; 电击形成的小孔会随着 膜磷脂的流动性而在数分钟内自行修复。这种技术可以将核苷酸、DNA 与 RNA、 蛋白、糖类、染料及病毒颗粒等导入原核和真核细胞内。相对其他物理和化学转化 方法, 电转化操作简便、瞬时高效、无需外源性转染试剂。电击过程中, 电荷在微 囊泡表面形成的间隙约为 $10 \mathrm{~nm}$, 因此要求用于标记的材料直径应该小于该阈值。

2. 标记材料的电荷、仪器工作电压等对标记效率有重要影响, 因此在实验中应予以考 虑并做调整优化。

3. 研究显示, 微囊泡表面在电荷作用下形成孔隙后, 标记材料可通过自由扩散的方式 进入微囊泡，因此在推荐允许的情况下，可以适当提高标记材料的浓度。

4. 操作过程中应使用仪器配套的电穿孔缓冲液, 以保证电穿孔的溶液的导电性。

5. 流式细胞仪对 EVs 的检测尤为关键, 普通流式检测检测下限为 200 500 nm, 无法 检测小的微囊泡及外泌体 $(30 \sim 150 \mathrm{~nm})$, 因此在分析此类微粒时, 目前有通过紫光 侧向角散射光替代前向散射光检测以及其他超高灵敏流式检测装置的流式细胞仪。

6. 实验中, 为排除杂质及噪音信号的影响, 使用的耗材一定要洁净, 缓冲液一定要经 $0.04 \mu \mathrm{m}$ 滤器过滤, 仪器的气路和液路尽量分别装上 $0.01 \mu \mathrm{m}$ 的空气过滤器和 0.04 $\mu \mathrm{m}$ 的鞘液过滤器。

\section{溶液配方}

1. 磷酸缓冲液 $(1 \times \mathrm{PBS})$

$0.24 \mathrm{~g}$ 磷酸二氢钾 $\left(\mathrm{KH}_{2} \mathrm{PO}_{4}\right)$

\section{$1.44 \mathrm{~g}$ 磷酸氢二钠 $\left(\mathrm{Na}_{2} \mathrm{HPO}_{4}\right)$}

$8 \mathrm{~g}$ 氯化钠 $(\mathrm{NaCl})$

$0.2 \mathrm{~g}$ 氯化钾 $(\mathrm{KCl})$

加去离子水约 $0.8 \mathrm{~L}$ 充分搅拌溶解, 然后加入浓盐酸调 $\mathrm{pH}$ 至 7.4 定容到 $1 \mathrm{~L}$

2. $\mathrm{NaOH}(0.1 \mathrm{M})$ 溶液 
用电子天平称取 $4 \mathrm{~g}$ 左右的 $\mathrm{NaOH}$, 置于小烧杯中, 用去离子水溶解后定容至 $1 \mathrm{~L}$ 容量瓶中，振荡摇勺

\section{致谢}

本文实验方案改编自武汉大学口腔医院余自力博士发表的论文，所述微囊泡由余博士提 供，相关流式细胞检测方法由中科院武汉病毒所分析测试中心建立并完成。

\section{参考文献}

1. Kanada, M., Bachmann, M. H., Hardy, J. W., Frimannson, D. O., Bronsart, L., Wang, A., Sylvester, M. D., Schmidt, T. L., Kaspar, R. L., Butte, M. J., Matin, A. C. and Contag, C. H. (2015). Differential fates of biomolecules delivered to target cells via extracellular vesicles. Proc Natl Acad Sci U S A 112(12): E1433-1442.

2. Pitt, J. M., Kroemer, G. and Zitvogel, L. (2016). Extracellular vesicles: masters of intercellular communication and potential clinical interventions. J Clin Invest 126(4): 1139-1143.

3. Resch-Genger, U., Graboolle, M., Cavaliere-Jaricot, S., Nitschke, R. and Nann, T. (2008). Quantum dots versus organic dyes as fluorescent labels. Nat Methods 5(9): 763-775.

4. Yu, Z. L., Zhang, W., Zhao, J. Y., Zhong, W. Q., Ren, J. G., Wu, M., Zhang, Z. L., Pang, D. W., Zhao, Y. F. and Chen, G. (2017). Development of a Dual-Modally Traceable Nanoplatform for Cancer Theranostics Using Natural Circulating Cell-Derived Microparticles in Oral Cancer Patients. Adv Funct Mater 27(40): 1703482. 